\title{
Effect of Presenilin Mutations on APP Cleavage; Insights into the Pathogenesis of FAD
}

\author{
Nuomin Li, Kefu Liu, Yunjie Qiu, Zehui Ren, Rongji Dai, Yulin Deng and Hong Qing* \\ School of Life Science, Beijing Institute of Technology, Beijing, China
}

Alzheimer disease (AD) is characterized by progressive memory loss, reduction in cognitive functions, and damage to the brain. The $\beta$-amyloid precursor protein can be sequentially cleaved by $\beta$ - secretase and $\gamma$-secretase. Mutations in the presenilin1(PS1) are the most common cause of Familial Alzheimer's disease (FAD). PS1 mutations can alter the activity of $\gamma$-secretase on the cleavage of the $\beta$-amyloid precursor protein, causing increased $A \beta$ production. Previous studies show that the $\beta A P P-C$-terminal fragment is first cleaved by $\beta$-scretase, primarily generating long fragments of $A \beta 48$ and $A \beta 49$, followed by the stepwise cleavage of every three amino acid residues at

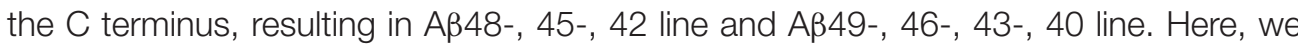
used LC-MS/MS to analyze unique peptides IAT, WIA, ITL, TVI, IVI through sequential cleavage, combined with ELISA to test the level of $A \beta 42$ and $A \beta 40$ for validation. The results show that most FAD mutant PS1 can alter the level of $A \beta 42$ and $A \beta 40$ monitored by the A $\beta 42 / A \beta 40$ ratio. Among them, six mutants (I143T, H163P, S170F, Q223R,

OPEN ACCESS

Edited by:

Roxana Octavia Carare, University of Southampton, UK

Reviewed by:

José M. Delgado-García, Pablo de Olavide University, Spain Robert Petersen, Case Western Reserve University, USA

*Correspondence: Hong Qing hqing@bit.edu.cn

Received: 05 January 2016 Accepted: 26 February 2016 Published: 11 March 2016

Citation:

Li N, Liu K, Qiu Y, Ren Z, Dai R, Deng $Y$ and Qing $H$ (2016) Effect of Presenilin Mutations on APP Cleavage; Insights into the Pathogenesis of FAD.

Front. Aging Neurosci. 8:51. doi: 10.3389/fnagi.2016.00051 $\mathrm{M} 233 \mathrm{~V}$, and $\mathrm{G} 384 \mathrm{~A})$ affect the $\mathrm{A} \beta 42 / 40$ ratio through both $\mathrm{A} \beta 49-40$ and $A \beta 48-38$ lines; L166P through decreasing the A $349-40$ line, six mutants (I143V, M146V, G217A, E280A, L381V, and L392V) through increasing the A $348-42$ line. More importantly, we found some mutations can affect the $\gamma$-secretase cleavage preference of $\alpha$-CTF and $\beta$-CTF. In conclusion, we found that the FAD PS1 mutations mainly increase the generation of $A \beta 42$ by decreasing the cleavage of $A \beta 42-A \beta 38$ and $A \beta 43-A \beta 40$.

Keywords: Familial Alzheimer's disease, $\gamma$-secretase, presenilin1, amyloid $\beta$-peptide

\section{INTRODUCTION}

Alzheimer disease $(\mathrm{AD})$ is the most common neurodegenerative disease. One of the main etiological hallmarks of $\mathrm{AD}$ is excessive production of $\mathrm{A} \beta$ (Bentahir et al., 2006; Cacquevel et al., 2012). $A \beta$ peptides aggregate and deposit into soluble oligomers, fibrils, and senile plaques, which are closely associated with synaptic dysfunction and neuronal network perturbations, finally causing gross atrophy of the brain (Lesne et al., 2006; Shankar et al., 2008; Sun et al., 2015). $\mathrm{A} \beta$ is a $38 \sim 43$ amino acid peptide derived from the $\beta$-APP through sequential cleavage by $\beta$-secretase (BACE1) and $\gamma$-secretase (Adlard et al., 2008; Thinakaran and Koo, 2008; Barnwell et al., 2014). BACE1 is an aspartyl protease $\beta$-site APP cleaving enzyme1 that cleaves APP mainly at a unique site, whereas the $\gamma$-secretase complex cleaves the CTF at several sites,

Abbreviations: A $\beta$, Amyloid $\beta$-peptide; APP, amyloid precursor protein; CTF, carboxy-terminal fragments; FAD, Familial Alzheimer's disease; PS1, Presenilin1; TMD, transmembrane domains. 
with preference for positions 40 and 42 , forming the $A \beta_{1-40}$ and $\mathrm{A} \beta_{1-42}$ peptides (Jonsson et al., 2012). Previous studies indicated that compared to other forms, such as $A \beta_{1-4}, A \beta_{1-38}, A \beta_{1-42}$, and $A \beta_{3-40}$ are more amyloidogenic (Chow et al., 2010). In particular, $A \beta 42$, which is more prone to aggregate and form soluble oligomers that eventually form insoluble plaques, is more amyloidogenic (Kakuda et al., 2006; Czirr et al., 2008). Clinical studies indicated that $A \beta_{1-42}$ peptide showed a higher percentage concentration in AD patients (Burdick et al., 1992; Kim et al., 2007). In the physiological condition, more than $90 \%$ of $A \beta$ is a shorter form of $A \beta 40$ and less than $5 \%$ of $A \beta$ is a longer form of A $\beta 42$ (Sun et al., 2015).

Autosomal dominant FAD is a rare form of $\mathrm{AD}$ and usually presents before the age of 65 years in individuals with a positive family history in at least three generations (Wu et al., 2012). Currently, more than 185 mutations have been identified in PS1, 13 in presenilin 2 (PS2) and 33 in APP among FDA patients (Cacquevel et al., 2012). PS1 is a trans-membrane protein that is an important component of the catalytic core of $\gamma$-secretase (Fernandez et al., 2014). $\gamma$-secretase, which is a multiprotein complex, is an unusual intramembranous cleaving aspartyl protease composed of presenilin, Nicastrin, Pen-2 and Aph-1 (Yu et al., 2000; Francis et al., 2002; Goutte et al., 2002; Christensen et al., 2004). PS1 is a highly conservative membrane protein, with nine TMDs. Large numbers of pathogenic mutations have been found throughout the coding sequence of PS1. Most of the PS1 mutations in FAD are located at TMDs (Wanngren et al., 2014), causing an increase in the $A \beta 42 / A \beta 40$ ratio, either by decreasing the production of $A \beta 40$ or increasing the production of $A \beta 42$ (Bentahir et al., 2006; Shen and Kelleher, 2007; Kretner et al., 2011). Other PS1 mutations in FAD, such as mutations at D257A and D385A are dominant negative, can lead to decreased $A \beta$ peptide secretion and the accumulation of the C-terminal fragments of the precursor protein (Kim et al., 2001). Previous studies showed that through $\gamma$-secretase, $\beta$ APP$\mathrm{C}$-terminal fragment $(\beta-\mathrm{CTF})$ is cleaved at the $\varepsilon$-site, generating primarily long fragments. Meanwhile, $A \beta 48$ and $A \beta 49$ is followed by stepwise cleavage of every three amino acid residues at the $\mathrm{C}$ terminus (Qi-Takahara et al., 2005). These findings led to the hypothesis that there are two $A \beta$ product lines: $A \beta 40$ and $\mathrm{A} \beta 42$. In this hypothesis, the $\mathrm{A} \beta 40$ product line represents the amino-terminal APP intracellular domain (AICD) 50-99 and $A \beta 49, A \beta 46, A \beta 43, A \beta 40$, and, the $A \beta 42$ product line represents AICD 49-99 and A $\beta 48, A \beta 45, A \beta 42, A \beta 38$ (He et al., 2010).

The PS1 gene has been widely studied since the discovery of FAD. While initial studies indicated the role of PS1 mutations in increased $\mathrm{A} \beta 42$ production in $\mathrm{FAD}$, it has now become clear that a series of pathogenic mutations caused impairments in other PS activities as well, such as A $\beta 40$, AICD, NICD and so on. Some researchers even proposed that pathogenic mutations in PS might play a role in the impaired $\gamma$-secretase-dependent and $\gamma$-secretase-independent activities through a dominant-negative mechanism. However, the molecular mechanisms remain elusive for FAD. Both D257A NTF and D385A CTF have been shown to abolish the $\gamma$-secretase activity in wild type or pathogenic PS1 mutants (Kim et al., 2005). Other mutations have also been shown to affect the $A \beta 42 / A \beta 40$ ratio: eight mutants (I143T, E280A, P284L, $\Delta$ exon9, G384A, F386S, S390I, $\mathrm{L} 392 \mathrm{P}$ ) were found to increase $\mathrm{A} \beta 42$, eleven (I143T, L166P, A246E, L250S, E280A, P284L, $\Delta$ exon9, P377M, G384A, L392V) decrease A $\beta 40$, and twelve (I143T, L166P, A246E, E280A, P284L, $\Delta$ exon9, R377M, G384A, F386S, S390I, L392P, L392V) decrease $A \beta 38$. These results show that decreased $A \beta 38$ and $A \beta 40$ and increased $\mathrm{A} \beta 42$ production are common phenotypes of PS1 mutations in FAD. Still more mutations were reported, but their exact role in FAD are still unclear (Houlden et al., 2001; Piccini et al., 2007; Uttner et al., 2010; Kim et al., 2012).

Given the important role the $A \beta 42 / A \beta 40$ ratio played in $\mathrm{AD}$, it is very important to understand the mechanism that leads to this change for the study of amyloidosis processing and AD onset (Kim et al., 2007). Here, we studied 13 different FAD PS1 mutations, plus one dominant negative mutation that affects the production of $A \beta$ through APP processing. We also quantitatively analyzed the triple and tetra peptide produced by two distinct lines of long amyloid $\beta$ cleavage processes with mass spectrometry. Our studies indicate that different mutations affect the $A \beta 42 / A \beta 40$ ratio through different mechanisms. Some decrease the cleavage of $A \beta 42$ to 38 (VVIA), while others decrease the cleavage of $A \beta 43$ to A $\beta 40$ (IAT). Such results can help us to better understand the underlying mechanism of PS mutations during the onset of $\mathrm{AD}$.

\section{MATERIALS AND METHODS}

\section{DNA Constructs and Mutagenesis}

BACE1-myc-his, pcC99, pcC83, wild-type PS1 human cDNAs (PS1-WT) were obtained from Weihong Song lab (University of British Columbia, Vancouver, BC, Canada). Mutations in PSEN1, (namely, I143T, I143V, M146V, H163P, L166P, S170F, G217A, M233V, Q223R, E280A, L381V, G384A, D385A, and $\mathrm{L} 392 \mathrm{~V}$ ) were generated by overlap extension PCR on the plasmid pcDNA4.1/PS1-WT using corresponding primers (Supplementary Table S1). The PCR fragments were then digested using EcoRI /HindIII, and subcloned into pcDNA4.1.

\section{Cell Culture and Transfection}

Human embryonic kidney 293 (HEK 293) cells, stably expressing "Swedish" mtAPP695 and BACE1 (2EB2 cell line), were cultured in Dulbecco's Modified Eagle Media, which is a Nutrient Mixture F-12 (GIBCO, CA) supplemented with $10 \%$ fetal bovine serum (FBS; GIBCO) and 1\% penicillin/streptomycin (GIBCO). Stable cell lines were selected using $200 \mathrm{cug} / \mathrm{ml}$ Zeocin and G418 (Invitrogen). Human embryonic kidney 293 (HEK 293) cells were cultured in Dulbecco's Modified Eagle Media (GIBCO, CA) supplemented with 10\% FBS (GIBCO) and 1\% penicillin/streptomycin (GIBCO). The cDNA constructs were transiently transfected into the cells using the Lipofectamine 2000 reagent (Invitrogen), according to the manufacturer's instructions. 


\section{Extraction of Tri-, Tetra-, and Pentapeptides from Living Cultured Cells}

2EB2 cells were transfected with PS1 or PS1 with various mutations. HEK293 cells co-transfected with pcC99 or pcC83 and PS1 or PS1 with various mutations were cultured to confluence in $10 \mathrm{~cm}$ dishes. Protease inhibitors (Protease inhibitor Cocktail Tablets, Roche, 04693132001) and $1 \mathrm{mM}$ 4-(2-Aminoethyl) benzenesulfonyl fluoride hydrochloride (AEBSF, sigma, A8456) were added into the conditioned medium $44 \mathrm{~h}$ after transfection. The cells were washed rapidly with ice-cold PBS, and then, immediately boiled for $2 \mathrm{~min}$. The boiled samples were sonicated for $3 \mathrm{~min}$ and centrifuged. The supernatant was then concentrated using a speed vacuum concentrator, and finally, subjected to an LCMS/MS analysis of the tripeptide and tetrapeptide (Okochi et al., 2013).

\section{A $\beta$ ELISA}

The conditioned medium was collected for an $\mathrm{A} \beta$ ( $\mathrm{A} \beta 40$ and $\mathrm{A} \beta 42$, Invitrogen) level assay. Protease inhibitor and 4-(2Aminoethyl) benzenesulfonyl fluoride hydrochloride were added to the conditioned medium $4 \mathrm{~h}$ before collection to prevent degradation of $A \beta$. The levels of $A \beta$ species ( $A \beta 40$ and $A \beta 42$ ) were measured by ELISA Kits according to the manufacturer's instructions.

\section{Identification and Quantification of Cleavage Peptides by LC-MS/MS}

An electrospray ionization tandem quadruple mass spectrometer, (Agilent 6460, USA) accompanied by ultra-performance liquid chromatography (Agilent 1260), was used to identify and quantify the cleavage peptides. Samples were maintained at $4{ }^{\circ} \mathrm{C}$ in the auto sampler. To quantify each peptide, a combination of precursor ion product ion pair was monitored using multiple reaction monitoring (MRM) modes. MRM methods were measured by LC-MS/MS as described previously (Takami et al., 2009). The $\mathrm{m} / \mathrm{z}$ values for these peptides were as follows: 502.7 and 199.2 for VVIAT; 425.7 and 261.2 for FLF; 345.8 and 215.1 for ITL; 329.8 and 185.2 for VIV; 303.7 and 185 for IAT; 331.8 and 185.1 for VIT; 331.8 and 173.1 for TVI; 401.2 and 171.1 for VVIA.

\section{Immunoblotting}

Cells were lysed $48 \mathrm{~h}$ after transfection in a RIPA Lysis Buffer with $50 \mathrm{mM}$ Tris- $\mathrm{HCl}$ (pH 7.4), $150 \mathrm{mM} \mathrm{NaCl}$, $1 \%$ NP-40, $0.1 \%$ SDS, Protease Inhibitor cocktail (Roche) and AEBSF. Sonication was done using an Ultrasonic Cell Disruptor (Sonics). The lysates were centrifuged at $14000 \mathrm{~g}$ for $10 \mathrm{~min}$ at $4^{\circ} \mathrm{C}$. The protein levels were determined by the Quick Start ${ }^{\mathrm{TM}}$ Bradford protein assay (Bio-Rad, 5000201). Cell lysate was subjected to SDS polyacrylamide gel electrophoresis (SDS-PAGE) with 16\% Tris-tricine (Li et al., 2006; Schagger, 2006) or 10\% Tris-glycine gels. The samples were then transferred to PVDF membranes (Millipore, $0.22 \mu \mathrm{m}$ ). For protein detection, membranes were blocked for $1 \mathrm{~h}$ with $5 \%$ milk, as well as incubated with the polyclonal C20 antibody against the last $20 \mathrm{C}$-terminus of human APP. The blots were developed using an ECL system and intensities of the bands were quantified with Image Lab ${ }^{\mathrm{TM}}$ Software (BioRad).

\section{Statistical Analysis}

Quantifications were done from data generated during three independent experiments. Values represent mean \pm standard error of the mean. Comparisons of more than two groups were carried out using one-way ANOVA and Dunnett's post hoc test using PS1 WT values as the control group (Fernandez et al., 2014). Statistical significance between the two groups was determined by an unpaired two-tailed $t$-test. $P<0.05$ was considered to be statistically significant.

\section{RESULTS}

\section{Human FAD PS1 Mutations Increase the Production of CTF}

In order to better understand the mechanisms of FAD PS1 mutations, 14 different PS1 mutations were selected and transiently transfected into $2 \mathrm{~EB} 2$ cells that stably overexpressed Swedish APP and BACE1 (Qing et al., 2004). PS1 has nine TMDs and harbors the catalytic site with two conserved aspartate residues located in TMD6 and TMD7 (Wolfe et al., 1999; Henricson et al., 2005; Spasic et al., 2006). It was reported that most PS1 mutations are located in TMD2, TMD3, TMD4, TMD5, TMD6, and TMD7. Among them, TMD1-6 and TMD8-9 are hydrophobic. TMD7 has a partial hydrophilic catalytic cavity and is very sensitive to mutations, which dramatically reduce its capability to insert into the cell membrane (Wanngren et al., 2014). TMD7 is part of the hydrophilic catalytic cavity, which is inserted in the hydrophobic core of the membrane, and probably protected by stable hydrophobic domains that include TMD1-6 and TMD8-9. TMD6 is also susceptible to changes in amino acid residues. Fourteen different FAD PS1 mutations that we selected are located in TMDs. Four are located in TMD2 (I143T/V, M146V, H163P); one in TMD3 (L166P, S170F); one in TMD4 (G217A), two in TMD5 (Q223R, M233V); one in H7 (E280A); and, three in TMD7 (L381V, L392V, G384A). One mutant (D385A) was also used as a negative control (Uttner et al., 2010). Except for L381V and G384A that have been well-studied, the rest have rarely, if ever, before been studied. Vector alone, PS1 WT (wild type) or PS1 mutants, were transiently transfected into $2 \mathrm{~EB} 2$ cells. Forty-eight hours after transfection, cells were collected, lysed, and a Western Blot was used to analyze the expression level of APP and CTFs, especially CTF99 expression. The levels of PS1 expression were used to verify the transfection efficiency. Compared with the vector alone (pcDNA4.1), both WT PS1 and PS1 with mutations could be transfected with high efficiency into the 2EB2 cell line. While the negative control mutation (D385A) did not affect the level of APP CTF expression, others, especially I143T, S170F, $\mathrm{M} 233 \mathrm{~V}$, and L392V, increased the expression of APP CTF (Figures 1A-C). 
A

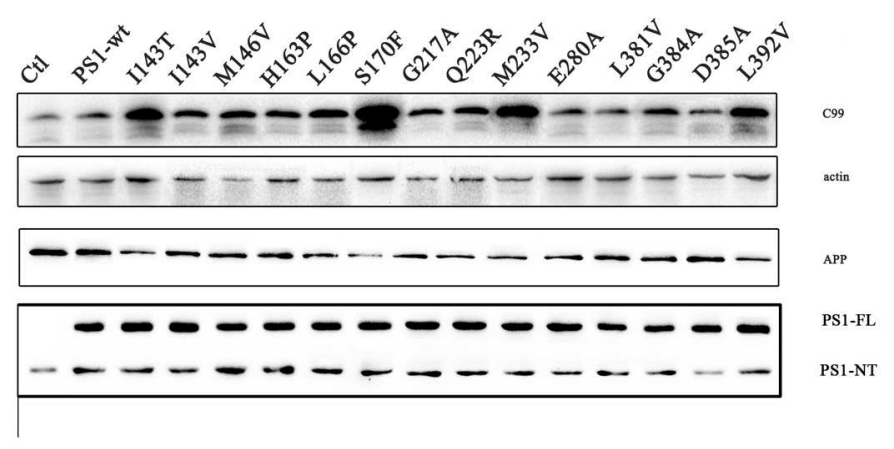

B

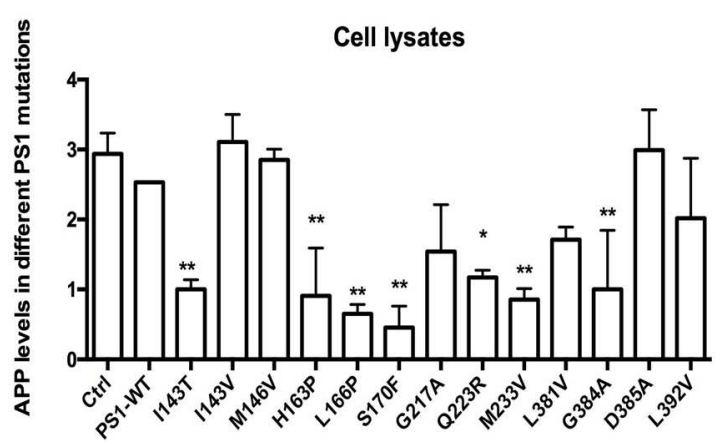

C

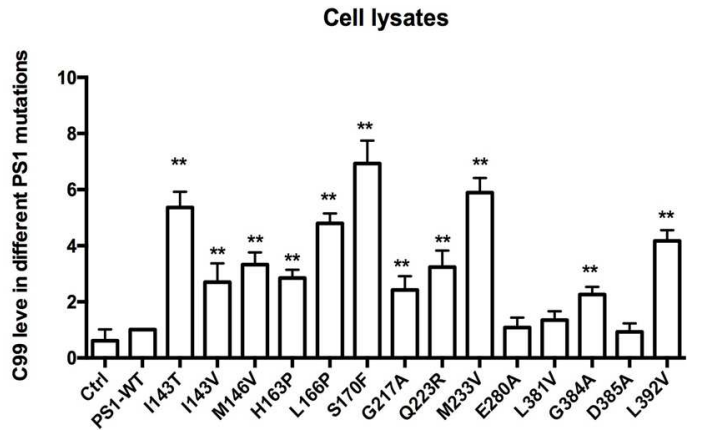

FIGURE 1 | Familial Alzheimer's disease PS1 mutations affect CTF level. Expression levels of APP, CTF in $2 E B 2$ cells expressing empty vector, PS1 WT, I143T, I143V, M146V, H163P, L166P, S170F, G217A, Q223R, M233V, E280A, L381V, G384A, D385A, L392V. (A) Cell lysates from human Swedish mutant APP695 and BACE1 double expression stable cell line 2EB2 were analyzed by Western Blot. APP, APP CTFs were detected with the C20 polyclonal antibody, PS1-NT was detected with the rat anti-presenilin-1 monoclonal antibody (Millipore, MAB1563). (B) Quantification of APP in 2EB2 cells in (A). (C) Quantification of C99 in 2EB2 cells in (A). ${ }^{*} P<0.05 ; * * P<0.01$.
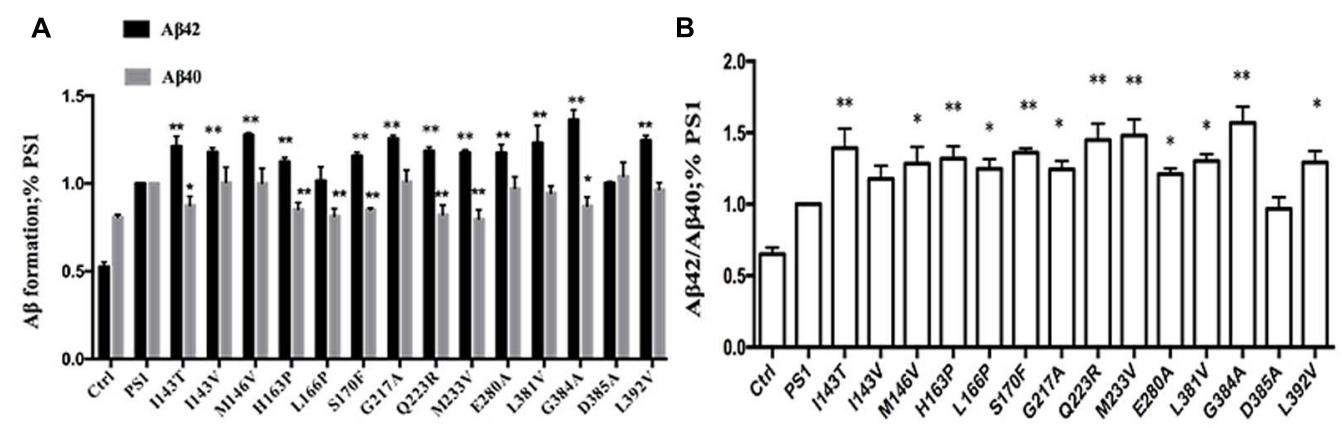

FIGURE 2 | Effects of PS1 WT and PS1 Mutants on long A $\beta$ Cleavage in 2EB2 cell line by ELISA (A,B). Conditioned medium from 2EB2 cells expressing

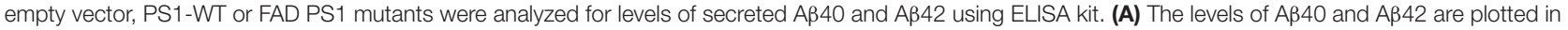
relative to PS1 WT. (B) The A $342 / A \beta 40$ ratio. ${ }^{*} P<0.05 ;{ }^{* *} P<0.01$.

\section{Human FAD PS1 Mutations Influence APP Cleavage Process}

To investigate the effect of human FAD PS1 on the APP cleavage process, empty vector (pcDNA4.1), PS1 WT or PS1 mutants were again transiently transfected into 2EB2 cells. Forty-eight hours after transfection, both the conditional medium and the cells were collected. ELISA was used to measure the levels of $\mathrm{A} \beta 42$ and $\mathrm{A} \beta 40$ in the conditional medium. Cells were lysed and the small peptides (tripeptide and tetrapeptide) were measured using LC-MS/MS (Agilent 6460, USA). ELISA results showed that compared to PS1 WT, the dominant negative mutation (D385A) did not affect the expression levels of $A \beta 42$ and $A \beta 40$, while most FAD PS1 mutants showed an increased $A \beta 42 / A \beta 40$ ratio through different lines. For example, L166P increased the ratio of $A \beta 42 / A \beta 40$ by lowering the level of $A \beta 40$. I143V, M146V, G217A, E280A, L381V, and L392V increased the A $\beta 42 / A \beta 40$ ratio through increased expression of $A \beta 42$. The rest of the mutations increased the ratio of $A \beta 42 / A \beta 40$ through both decreasing $A \beta 40$ 
and increasing $A \beta 42$ levels at the same time (Figures 2A,B). The amounts of tripeptide and tetrapeptide produced during the stepwise processing of longer form $A \beta$ in living cells were then measured (Figures 3 and 4 ). The result indicated that neither the negative control nor any of the selected FAD PS1 mutations affected the selectivity of the $\gamma$-secretase cleavage of long form $A \beta$, judged by the total level of $A \beta 49-40$ relative to that of total $\mathrm{A} \beta$-related small peptides (Figure 4A). Compared to the PS1-WT, except for L166P and D385A mutations, most PS1 mutations were able to reduce the relative rate of $A \beta 42$ cleavage into $A \beta 38$. This reduction was concluded based upon the level of VVIA relative to that of total $A \beta 48-42$ related small peptides (Figure 4B). Correspondingly, compared with PS1WT, most FAD PS1 mutations (I143T, H163P, L166P, S170F, Q223R, M233V, E280A, and G384A) were able to decrease the relative rate of $A \beta 43$ cleavage into $A \beta 40$. This finding is based upon the level of IAT relative to that of the total A $349-40$ related small peptides (Figure 4C). The LC-MS/MS results are consistent with the results from ELISA and Western Blot.
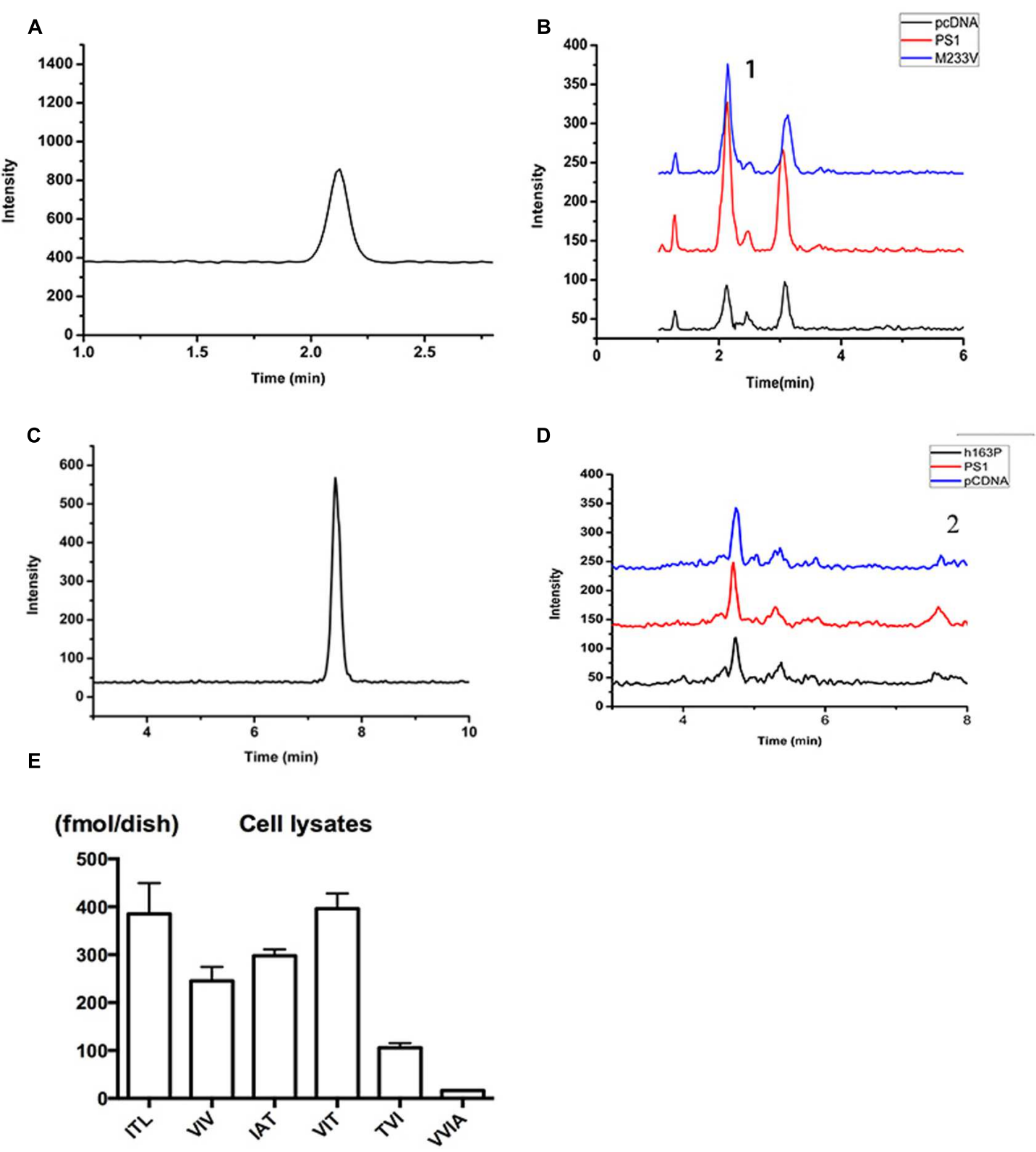

FIGURE 3 | Detection of related small peptide species of A $\boldsymbol{\beta}$. (A) The chromatographys of IAT standard peptides. (B) The Intensity of multiple reactions monitoring (MRM) ion chromatography of ITA. (1) Its transition is $\mathrm{m} / \mathrm{z}=303.7 / 185$. Black, pCDNA4.1; Red, PS1; Blue, M233V. (C) The chromatographys of WIA standard peptides. (D) The Intensity of multiple reactions monitoring (MRM) ion chromatography of WIA. (2) Its transition is m/z = 401.2/171.1. Black, pCDNA4.1; Red, PS1; Blue, M233V. (E) A $\beta$ species in lysates of 2EB2 cells in a $10 \mathrm{~cm}$ dish overexpressing PS1 WT. 


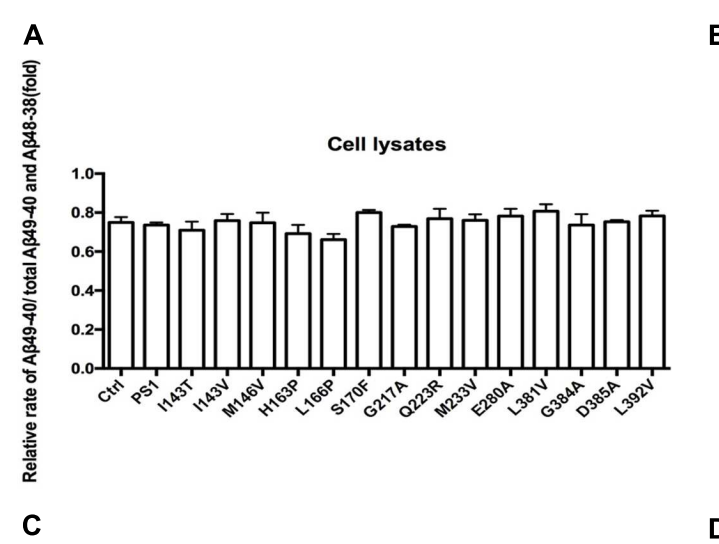

B

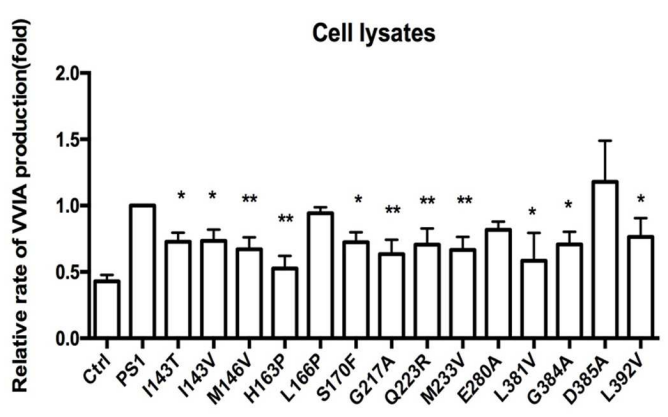

D
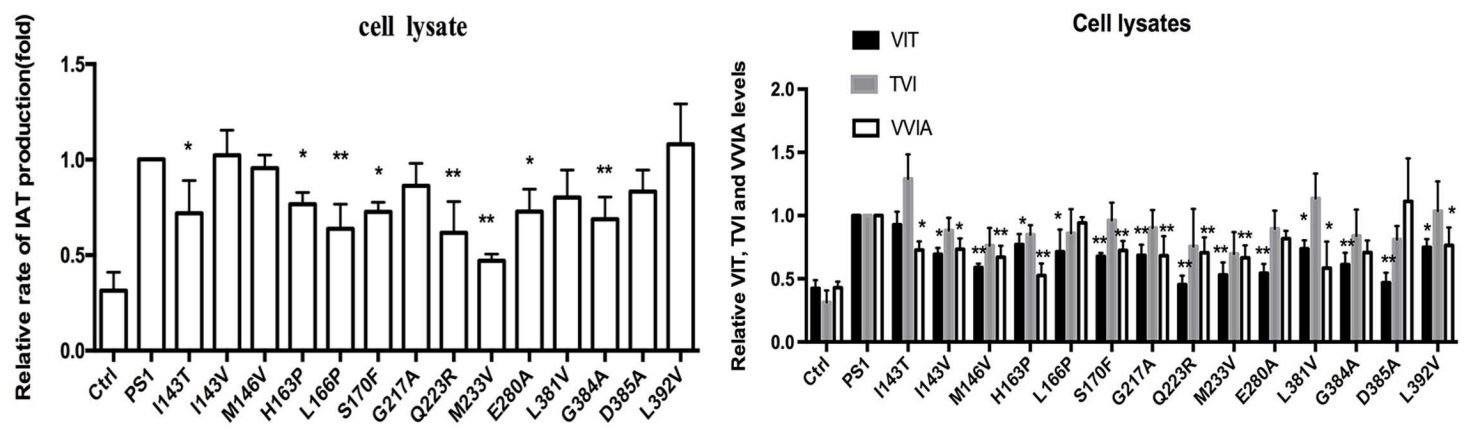

E

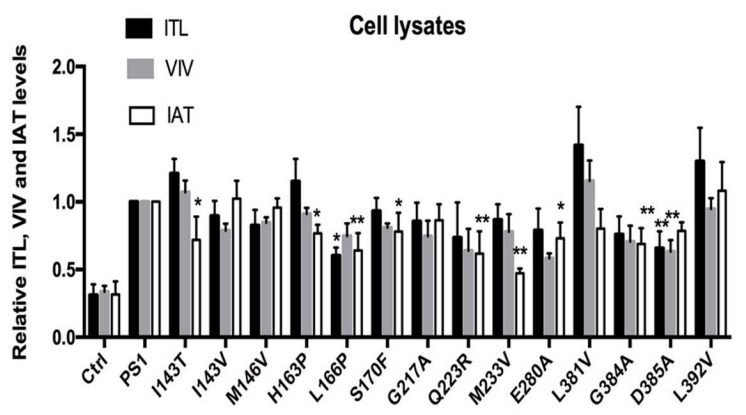

FIGURE 4 | Effects of PS-WT1 and PS1 Mutants on long A $\beta$ Cleavage in 2EB2 cell line by LC-MS/MS. (A) Fold changes of the relative rate of A $449-40 /$ total A $\beta$ production. (B) Fold changes of the relative VVIA levels in cell lysates of 2EB2 cell line. (C) Fold changes of the relative IAT levels in cell lysates of 2EB2 cells line. (D) Fold changes of the relative VIT, TVI, and VIA levels in cell line expressing PS1 and PS1 FAD mutants. (E) Fold changes of the relative ITL, VIV, and IAT levels in cell line expressing PS1 and PS1 FAD mutants. ${ }^{*} P<0.05 ;{ }^{*} P<0.01$.

\section{Human FAD PS1 Mutations Differ in Their Effect on $A \beta$ Generated by $\beta$-CTF Line}

Amyloid precursor protein can be cleaved into $\alpha$-CTF (CTF83) and $\beta$-CTF (CTF99) by $\alpha$-secretase and $\beta$-secretase in vivo, respectively. Therefore, we want to examine whether CTF83 can affect the CTF99 cleavage with PS1 mutations. pzC99, which is the cDNA encoding CTF99, was inserted into the pcDNA4.1 vector. We co-transfected transiently pzC99 with the empty vector, PS1 WT or PS1 mutants, into HEK293 cells. Fortyeight hours after transfection, both the condition medium and the cells were collected. The levels of $A \beta 42$ and $A \beta 40$ in the culture medium were similar to the previous ELISA results (Figures 2A,B). Most FAD PS1 mutations also increased the ratio of $A \beta 42 / A \beta 40$ as shown before: $L 166 \mathrm{P}$ increased the ratio of $A \beta 42 / A \beta 40$ by reducing $A \beta 40$ level; I143V, M146V, G217A, E280A, L381V, and G384 increased the ratio of $A \beta 42 / A \beta 40$ by increasing $A \beta 42$ level; and finally, the rest of the mutations increased the ratio of $A \beta 42 / A \beta 40$ by both reducing $A \beta 40$ and increasing A $\beta 42$ levels (Figures 5A,B). Cells were then lysed and the tripeptide and tetrapeptide were measured using LC-MS/MS (Figure 3). The results were in agreement with previous results in the A $\beta 48-A \beta 38$ line (Figure 4D). Compared with PS1-WT, some FAD PS1 mutations (I143T, I143V, M146V, H163P, S170F, G217A, Q223R, M233V, E280A, L381V, G384A, and L392V), showed elevated levels of $A \beta 42$ as a result of lower VVIA, which was produced by the cleavage of $\mathrm{A} \beta 42$ into $\mathrm{A} \beta 38, \mathrm{~L} 166 \mathrm{P}$ and 


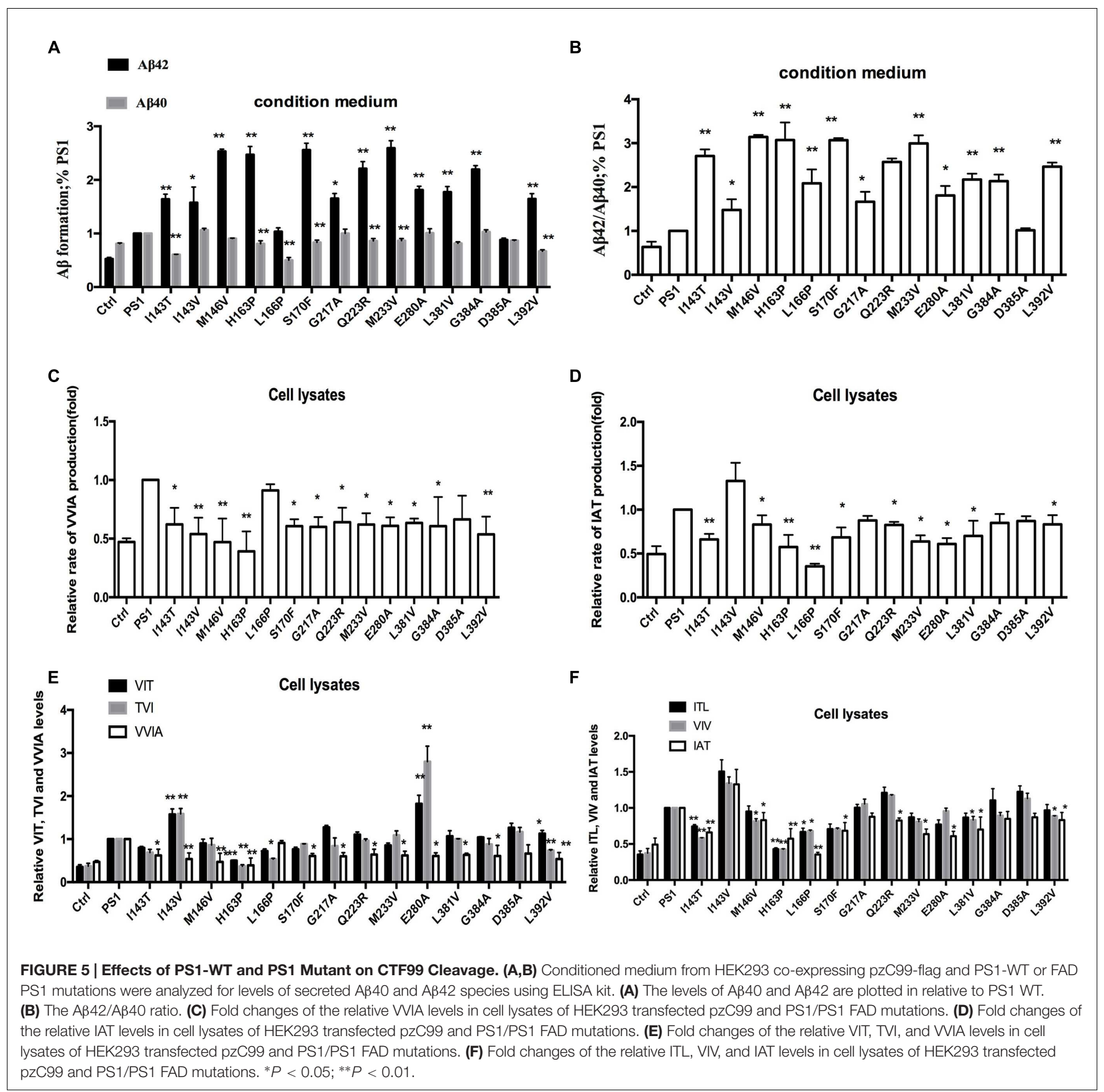

negative control had no effect on the VVIA level (Figure 5C). The results of $A \beta 49-A \beta 40$ line were slightly different (Figure 4E). Some FAD PS1 mutations (I143T, M146V, H163P, L166P, S170F, Q223R, M233V, E280A, L381V, and L392V) secreted lower levels of $A \beta 40$, causing a lower level of IAT to be generated by the cleavage of $A \beta 43$ into $A \beta 40$. Others, such as I143V, G217A, and G384A, as well as the negative control, did not affect the IAT levels (Figure 5D). Among them, M146V, G384A, L381V, and L392V showed slightly different results from the previous data (Figures 4B,C). This could be due to CTF83 competing with CTF99 for cleavage by $\gamma$-secretase.

\section{Human FAD PS1 Mutations Differ in Their Effect on $A \beta$ by $\alpha$-CTF Line}

The above results showed that FAD PS1 mutations differ in their effects on the A $\beta$ generation line of APP and CTF99, especially in the A $\beta 49-A \beta 40$ line. APP could be cleaved by different secretase to generate $\alpha$-CTF and $\beta$-CTF. Subsequently, they could be sequentially cleaved to produce $A \beta$ fragments using $\gamma$-secretase. Both $\alpha$-CTF and $\beta$-CTF can give rise to the tri- and tetra-peptides, but only $\beta$-CTF can eventually generate $A \beta 42$. Thus, we further tested the hypothesis that the presence of CTF83 may affect the cleavage of CTF99. 

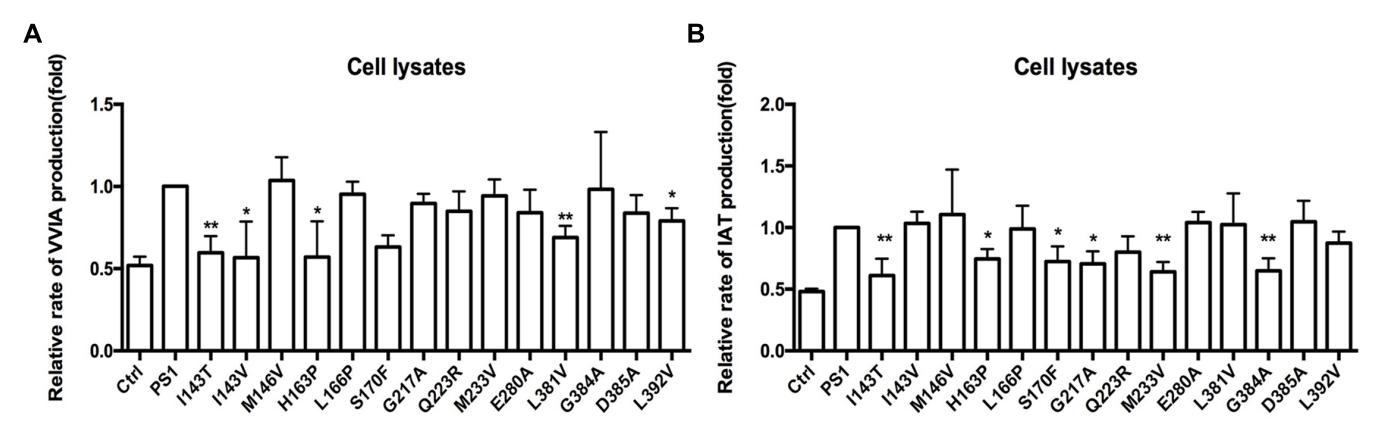

FIGURE 6 | Effects of PS1-WT and PS1 Mutants on CTF83 Cleavage. (A) Fold changes of the relative WIA levels in cell lysates of HEK293 transfected pzC83 and PS1-WT or FAD PS1 mutations. (B) Fold changes of the relative IAT levels in cell lysates of HEK293 transfected pzC83 and PS1-WT or FAD PS1 mutations. ${ }^{*} P<0.05 ;{ }^{* *} P<0.01$.

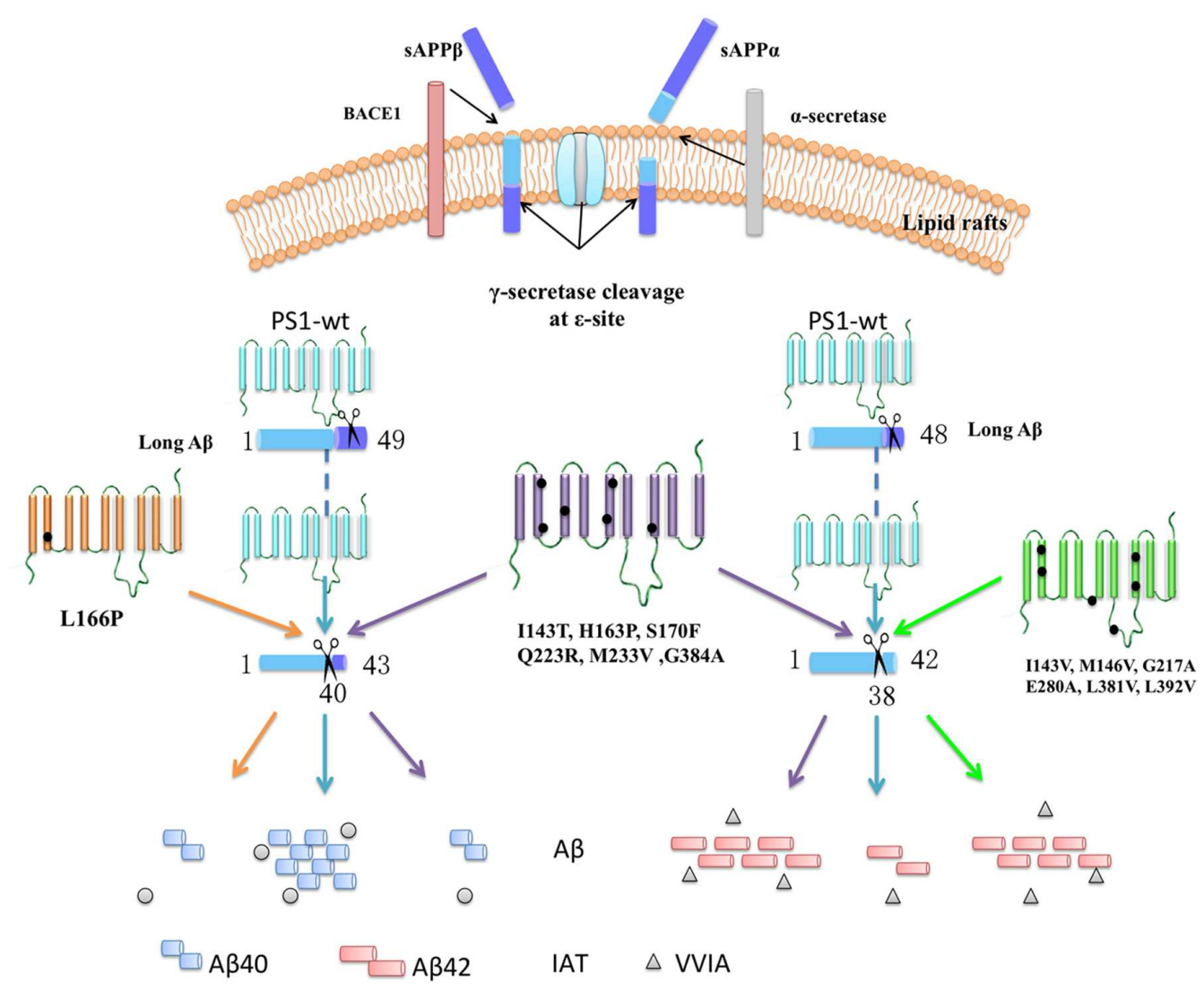

FIGURE 7 | Summarize the effects on specific peptides cleaved from long A $\beta$ in different mutations. The APP can be sequential cleavage by $\beta$-secretase (BACE1)/ $\alpha$-secretase and $\gamma$-secretase. APP-C-terminal fragment is cleaved at the $\varepsilon$-site by $\gamma$-secretase, generating primarily long fragments, A 348 and A 449 , followed by stepwise cleavage of every three amino acid residues at the $\mathrm{C}$ terminus, generating unique peptide. PS1 negative control, D385A, showed no change in unique peptide WIA and IAT. Six mutations (1143T, H163P, S170F, Q223R, M233V, and G384A) change the ratio of A $442 / 40$ by decreasing the cleavage of A $443-40$

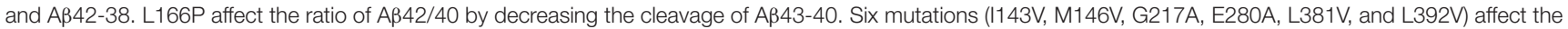
ratio of $A \beta 42 / 40$ by decreasing the cleavage of $A \beta 42-38$.

ELISA results showed that $\mathrm{A} \beta 42$ could not be detected in either PS1-WT or FAD PS1 mutations. The LC-MS/MS results showed that compared to PS-WT, some FAD PS1 mutations (I143T, I143V, H163P, S170F, L381V, and L392V) produced lower levels of VVIA (Figure 6A). Meanwhile, others (I143T,
H163P, S170F, G217A, M233V, and G384A) produced lower levels of IAT (Figure 6B). Combined with the data of a specific peptide IAT cleaved from APP and a $\beta$-CTF cleavage using $\gamma$-secretase (Figures 4 and 5), we propose that the existenceof $\alpha$-CTF may affect the $\beta$-CTF cleavage in certain, (i.e., M146V, 
L381V, G384A, and L392V) but not all, FAD PS1 mutations. Moreover, the presence of CTF83 may affect the cleavage of CTF99.

\section{DISCUSSION}

The PS1 mutation is known to be a key heredity factor of FAD. Mutation in PS1 can cause changes to $\gamma$-secretase activities, resulting in the alteration of the $A \beta 42 / 40$ ratio. Recent research data show that $A \beta$ can be generated by a series and continuous cleavage using $\gamma$-secretase (Takami et al., 2009; Okochi et al., 2013; Olsson et al., 2014). LC-MS/MS can be used to monitor the cleavage progress by following the tripeptides and tetrapeptides generated during the process. Based on the $A \beta 48-\mathrm{A} \beta 38$ and $\mathrm{A} \beta 49-\mathrm{A} \beta 40$ cleavage lines, 14 PS1 mutations impacting the $\mathrm{A} \beta 42 / 40$ ratio via different lines of $\mathrm{A} \beta$ generated line were reported. Here, we discovered four significant findings. First, the PS1 negative control (D385A) showed no change in $A \beta 42$, $\mathrm{A} \beta 40$ level, or $\mathrm{A} \beta 42 / 40$ ratio. This outcome is likely because they do not affect the cleavage of CTF83 and CTF99, especially the unique peptide VVIA and IAT. LC-MS/MS results indicated that they do not significantly affect the CTF83 and CTF99 cleavage line either. Second, six mutations (I143T, H163P, S170F, Q223R, $\mathrm{M} 233 \mathrm{~V}$, and $\mathrm{G} 384 \mathrm{~A}$ ) change the ratio of $\mathrm{A} \beta 42 / 40$ by decreasing the level of $A \beta 40$ and increasing the level of $A \beta 42$, respectively. Meanwhile, the cleavage of $A \beta 42$ to $A \beta 38$ and of $A \beta 43$ to $A \beta 40$ were both decreased. Such results indicate that those mutations affect the $A \beta 42 / 40$ ratio through both the $A \beta 49-40$ line and the $\mathrm{A} \beta 48-42$ line. Third, one mutation (L166P) affected the ratio of $A \beta 42 / 40$ by decreasing the level of $A \beta 40$ as a result of the decreased specific cleavage of $A \beta 43$ to $A \beta 40$, with no effect on $\mathrm{A} \beta 42$. This outcome indicates that L166P affects the $A \beta 42 / 40$ ratio mainly through the $A \beta 49-40$ line. Fourth, six mutations (I143V, M146V, G217A, E280A, L381V, and L392V) affected the ratio of $A \beta 42 / 40$ by increasing the level of $A \beta 42$, via specifically decreasing the cleavage of $A \beta 42$ to $A \beta 38$, with no effect on $A \beta 40$. This outcome suggests that those mutations affect the $A \beta 42 / 40$ ratio mainly through the $A \beta 48-A \beta 40$ line. To summarize, except for the negative control (D385A), which showed no effect on the $\mathrm{A} \beta 42 / 40$ ratio and the process of $\beta$-CTF cleavage, most of PS1 mutations could change the $\mathrm{A} \beta 42 / 40$ ratio through different long form $A \beta$ cleavage lines (Figure 7 ).

Exactly how FAD mutations lead to the AD neuropathogenesis is still a mystery at present. One prevailing hypothesis is that PS mutations in mammalian systems cause the increase of the $\gamma$-secretase activity and enhance $A \beta 42$ production (Shioi et al., 2007). Conversely, some new studies suggest that some mutations in FAD PS1 mutations (i.e., V82L, C263R et al.) not only would not increase production of $A \beta 42$, but also lead to a loss of its essential functions. Many studies showed that FAD PS1 mutations would increase the ratio of $A \beta 42 / A \beta 40$. However, previous studies primarily focus on, the lowered production of $\mathrm{A} \beta 40$, rather than the increased production of $\mathrm{A} \beta 42$ (Shen and Kelleher, 2007). Our data seems to be in agreeing with the previous hypotheses. Here, we show that L166p impacts the $A \beta 42 / A \beta 40$ ratio by decreasing the level of $A \beta 40$, rather than increasing the level of $A \beta 42$ corresponds with previous work. Three PS1 mutations (L133P, G183V, and insR352) were found to cause a lack of amyloid pathology (Raux et al., 2000; Amtul et al., 2002; Dermaut et al., 2004) and an absence of A $\beta$ accumulation. Such mutations are believed to be associated with Frontotemporal Dementia (FTD) more than with FAD, despite the fact they can be found in both FAD and FTD patients. The data we collected leads us to believe that most FAD PS1 mutations lead to amyloid pathogenesis. This outcome maybe due to FAD PS1 mutations function in regulating the cleavage of $A \beta 42-A \beta 38$ and $\mathrm{A} \beta 43-\mathrm{A} \beta 40$.

Interestingly, our results indicated that PS1 mutations have different effects on the $\beta$-CTF and APP processing of A $\beta$ generation. It is known that APP can be cleaved by $\beta$-secretase and $\alpha$-secretase, generating $\beta$-CTF and $\alpha$-CTF. Subsequently, $\alpha$-CTF and $\beta$-CTF may both be cleaved by $\gamma$-secretase. Additionally, $\alpha$-CTF may remain the same peptide through the processing of $\gamma$-secretase cleavage. Here, we detected tri-peptides and tetra-peptides in the HEK293 cell linings co-transfected with the PS1 mutation and CTF83. We found that $\alpha$-CTF is also processed by a series of continuous cleavages that produce the same tri-peptides and tetra-peptides as that of $\beta$-CTF. Moreover, the PS1 mutation could alter the cleavage process of $\alpha$-CTF as well. Some mutations have different effects on the processing of $\alpha$-CTF and $\beta$-CTF cleavages, suggesting that changes in the PS1 structure may decrease a $\beta$-CTF cleavage with no effect on $\alpha$-CTF.

\section{CONCLUSION}

Most mutations in PS1 accelerate the amyloid formation by affecting the $A \beta$ generation process that results in a change of $\mathrm{A} \beta 42 / 40$ in FAD causing dementia.

\section{AUTHOR CONTRIBUTIONS}

NL and YQ contributed to the cell culture and sample preparation. ZR contributed to the data analysis. KL contributed to the sample detection by LC-MS/MS. DR and YD contributed to the experimental design and discussion. NL and HQ contributed to study design and manuscript preparation.

\section{ACKNOWLEDGMENTS}

This study was funded by National Natural Science Foundation of China (No. 81171206) and partially supported by the ministry of Science and Technology, China (No. 2013YQ03059514) and a grant from the Key Laboratory for Neurodegenerative Disease of Ministry of Education (No. 2015SJBX05, 2015SJZS01).

\section{SUPPLEMENTARY MATERIAL}

The Supplementary Material for this article can be found online at: http://journal.frontiersin.org/article/10.3389/fnagi. 2016.00051 


\section{REFERENCES}

Adlard, P. A., Cherny, R. A., Finkelstein, D. I., Gautier, E., Robb, E., Cortes, M., et al. (2008). Rapid restoration of cognition in Alzheimer's transgenic mice with 8 -hydroxy quinoline analogs is associated with decreased interstitial Abeta. Neuron 59, 43-55. doi: 10.1016/j.neuron.2008.06.018

Amtul, Z., Lewis, P. A., Piper, S., Crook, R., Baker, M., Findlay, K., et al. (2002). A presenilin 1 mutation associated with familial frontotemporal dementia inhibits gamma-secretase cleavage of APP and notch. Neurobiol. Dis. 9, 269273. doi: $10.1006 /$ nbdi.2001.0473

Barnwell, E., Padmaraju, V., Baranello, R., Pacheco-Quinto, J., Crosson, C., Ablonczy, Z., et al. (2014). Evidence of a novel mechanism for partial gamma-secretase inhibition induced paradoxical increase in secreted amyloid beta protein. PLOS ONE 9:e91531. doi: 10.1371/journal.pone.00 91531

Bentahir, M., Nyabi, O., Verhamme, J., Tolia, A., Horre, K., Wiltfang, J., et al. (2006). Presenilin clinical mutations can affect gamma-secretase activity by different mechanisms. J. Neurochem. 96, 732-742. doi: 10.1111/j.14714159.2005.03578.x

Burdick, D., Soreghan, B., Kwon, M., Kosmoski, J., Knauer, M., Henschen, A., et al. (1992). Assembly and aggregation properties of synthetic Alzheimer's A4/beta amyloid peptide analogs. J. Biol. Chem. 267, 546-554.

Cacquevel, M., Aeschbach, L., Houacine, J., and Fraering, P. C. (2012). Alzheimer's disease-linked mutations in presenilin-1 result in a drastic loss of activity in purified gamma-secretase complexes. PLOS ONE 7:e35133. doi: 10.1371/journal.pone.0035133

Chow, V. W., Mattson, M. P., Wong, P. C., and Gleichmann, M. (2010). An overview of APP processing enzymes and products. Neuromol. Med. 12, 1-12. doi: 10.1007/s12017-009-8104-Z

Christensen, M. A., Zhou, W., Qing, H., Lehman, A., Philipsen, S., and Song, W. (2004). Transcriptional regulation of BACE1, the beta-amyloid precursor protein beta-secretase, by Sp1. Mol. Cell Biol. 24, 865-874. doi: 10.1128/MCB.24.2.865-874.2004

Czirr, E., Cottrell, B. A., Leuchtenberger, S., Kukar, T., Ladd, T. B., Esselmann, H., et al. (2008). Independent generation of Abeta42 and Abeta38 peptide species by gamma-secretase. J. Biol. Chem. 283, 17049-17054. doi: 10.1074/jbc.M802912200

Dermaut, B., Kumar-Singh, S., Engelborghs, S., Theuns, J., Rademakers, R., Saerens, J., et al. (2004). A novel presenilin 1 mutation associated with Pick's disease but not beta-amyloid plaques. Ann. Neurol. 55, 617-626. doi: 10.1002/ana.20083

Fernandez, M. A., Klutkowski, J. A., Freret, T., and Wolfe, M. S. (2014). Alzheimer presenilin-1 mutations dramatically reduce trimming of long amyloid betapeptides (Abeta) by gamma-Secretase to Increase 42-to-40-Residue Abeta. J. Biol. Chem. 289, 31043-31052. doi: 10.1074/jbc.M114.581165

Francis, R., McGrath, G., Zhang, J., Ruddy, D. A., Sym, M., Apfeld, J., et al. (2002). aph-1 and pen-2 are required for Notch pathway signaling, gamma-secretase cleavage of beta APP, and presenilin protein accumulation. Dev. Cell 3, 85-97. doi: 10.1016/S1534-5807(02)00189-2

Goutte, C., Tsunozaki, M., Hale, V. A., and Priess, J. R. (2002). APH-1 is a multipass membrane protein essential for the Notch signaling pathway in Caenorhabditis elegans embryos. Proc. Natl. Acad. Sci. U.S.A. 99, 775-779. doi: 10.1073/pnas.022523499

He, G., Luo, W., Li, P., Remmers, C., Netzer, W. J., Hendrick, J., et al. (2010). Gamma-secretase activating protein is a therapeutic target for Alzheimer's disease. Nature 467, 95-98. doi: 10.1038/nature09325

Henricson, A., Kall, L., and Sonnhammer, E. L. (2005). A novel transmembrane topology of presenilin based on reconciling experimental and computational evidence. FEBS J. 272, 2727-2733. doi: 10.1111/j.1742-4658.2005.04691.x

Houlden, H., Crook, R., Dolan, R. J., McLaughlin, J., Revesz, T., and Hardy, J. (2001). A novel presenilin mutation (M233V) causing very early onset Alzheimer's disease with Lewy bodies. Neurosci. Lett. 313, 93-95. doi: 10.1016/S0304-3940(01)02254-6

Jonsson, T., Atwal, J. K., Steinberg, S., Snaedal, J., Jonsson, P. V., Bjornsson, S., et al. (2012). A mutation in APP protects against Alzheimer's disease and age-related cognitive decline. Nature 488, 96-99. doi: 10.1038/nature11283

Kakuda, N., Funamoto, S., Yagishita, S., Takami, M., Osawa, S., Dohmae, N., et al. (2006). Equimolar production of amyloid beta-protein and amyloid precursor protein intracellular domain from beta-carboxyl-terminal fragment by gammasecretase. J. Biol. Chem. 281, 14776-14786. doi: 10.1074/jbc.M513453200

Kim, H., Ki, H., Park, H. S., and Kim, K. (2005). Presenilin-1 D257A and D385A mutants fail to cleave Notch in their endoproteolyzed forms, but only presenilin-1 D385A mutant can restore its gamma-secretase activity with the compensatory overexpression of normal C-terminal fragment. J. Biol. Chem. 280, 22462-22472. doi: 10.1074/jbc.M502769200

Kim, J., Bagyinszky, E., Chang, Y. H., Choe, G., Choi, B. O., An, S. S., et al. (2012). A novel PSEN1 H163P mutation in a patient with early-onset Alzheimer's disease: clinical, neuroimaging, and neuropathological findings. Neurosci. Lett. 530, 109-114. doi: 10.1016/j.neulet.2012.09.040

Kim, J., Onstead, L., Randle, S., Price, R., Smithson, L., Zwizinski, C., et al. (2007). Abeta40 inhibits amyloid deposition in vivo. J. Neurosci. 27, 627-633. doi: 10.1523/JNEUROSCI.4849-06.2007

Kim, S. H., Leem, J. Y., Lah, J. J., Slunt, H. H., Levey, A. I., Thinakaran, G., et al. (2001). Multiple effects of aspartate mutant presenilin 1 on the processing and trafficking of amyloid precursor protein. J. Biol. Chem. 276, 43343-43350. doi: 10.1074/jbc.M108245200

Kretner, B., Fukumori, A., Gutsmiedl, A., Page, R. M., Luebbers, T., Galley, G., et al. (2011). Attenuated Abeta42 responses to low potency gamma-secretase modulators can be overcome for many pathogenic presenilin mutants by second-generation compounds. J. Biol. Chem. 286, 15240-15251. doi: 10.1074/jbc.M110.213587

Lesne, S., Koh, M. T., Kotilinek, L., Kayed, R., Glabe, C. G., Yang, A., et al. (2006). A specific amyloid-beta protein assembly in the brain impairs memory. Nature 440, 352-357. doi: 10.1038/nature04533

Li, Y., Zhou, W., Tong, Y., He, G., and Song, W. (2006). Control of APP processing and Abeta generation level by BACE1 enzymatic activity and transcription. FASEB J. 20, 285-292. doi: 10.1096/fj.05-4986com

Okochi, M., Tagami, S., Yanagida, K., Takami, M., Kodama, T. S., Mori, K., et al. (2013). gamma-secretase modulators and presenilin 1 mutants act differently on presenilin/gamma-secretase function to cleave Abeta42 and Abeta43. Cell Rep. 3, 42-51. doi: 10.1016/j.celrep.2012.11.028

Olsson, F., Schmidt, S., Althoff, V., Munter, L. M., Jin, S., Rosqvist, S., et al. (2014). Characterization of intermediate steps in amyloid beta (Abeta) production under near-native conditions. J. Biol. Chem. 289, 1540-1550. doi: 10.1074/jbc.M113.498246

Piccini, A., Zanusso, G., Borghi, R., Noviello, C., Monaco, S., Russo, R., et al. (2007). Association of a presenilin 1 S170F mutation with a novel Alzheimer disease molecular phenotype. Arch. Neurol. 64, 738-745. doi: 10.1001/archneur.64.5.738

Qing, H., Zhou, W., Christensen, M. A., Sun, X., Tong, Y., and Song, W. (2004). Degradation of BACE by the ubiquitin-proteasome pathway. FASEB J. 18, 1571-1573. doi: 10.1096/fj.04-1994fje

Qi-Takahara, Y., Morishima-Kawashima, M., Tanimura, Y., Dolios, G., Hirotani, N., Horikoshi, Y., et al. (2005). Longer forms of amyloid beta protein: implications for the mechanism of intramembrane cleavage by gamma-secretase. J. Neurosci. 25, 436-445. doi: 10.1523/JNEUROSCI.1575-04.2005

Raux, G., Gantier, R., Thomas-Anterion, C., Boulliat, J., Verpillat, P., Hannequin, D., et al. (2000). Dementia with prominent frontotemporal features associated with L113P presenilin 1 mutation. Neurology 55, 1577-1578. doi: 10.1212/WNL.55.10.1577

Schagger, H. (2006). Tricine-SDS-PAGE. Nat. Protoc. 1, 16-22. doi: 10.1038 /nprot.2006.4

Shankar, G. M., Li, S., Mehta, T. H., Garcia-Munoz, A., Shepardson, N. E., Smith, I., et al. (2008). Amyloid-beta protein dimers isolated directly from Alzheimer's brains impair synaptic plasticity and memory. Nat. Med. 14, 837-842. doi: $10.1038 / \mathrm{nm} 1782$

Shen, J., and Kelleher, R. J. III. (2007). The presenilin hypothesis of Alzheimer's disease: evidence for a loss-of-function pathogenic mechanism. Proc. Natl. Acad. Sci. U.S.A. 104, 403-409. doi: 10.1073/pnas.0608332104

Shioi, J., Georgakopoulos, A., Mehta, P., Kouchi, Z., Litterst, C. M., Baki, L., et al. (2007). FAD mutants unable to increase neurotoxic Abeta 42 suggest that mutation effects on neurodegeneration may be independent of effects on Abeta. J. Neurochem. 101, 674-681. doi: 10.1111/j.1471-4159.2006.04391.x

Spasic, D., Tolia, A., Dillen, K., Baert, V., De Strooper, B., Vrijens, S., et al. (2006). Presenilin-1 maintains a nine-transmembrane topology 
throughout the secretory pathway. J. Biol. Chem. 281, 26569-26577. doi: 10.1074/jbc.M600592200

Sun, X., Chen, W. D., and Wang, Y. D. (2015). beta-Amyloid: the key peptide in the pathogenesis of Alzheimer's disease. Front. Pharmacol. 6:221. doi: 10.3389/fphar.2015.00221

Takami, M., Nagashima, Y., Sano, Y., Ishihara, S., Morishima-Kawashima, M., Funamoto, S., et al. (2009). gamma-Secretase: successive tripeptide and tetrapeptide release from the transmembrane domain of beta-carboxyl terminal fragment. J. Neurosci. 29, 13042-13052. doi: 10.1523/JNEUROSCI.236209.2009

Thinakaran, G., and Koo, E. H. (2008). Amyloid precursor protein trafficking, processing, and function. J. Biol. Chem. 283, 29615-29619. doi: 10.1074/jbc.R800019200

Uttner, I., Kirchheiner, J., Tumani, H., Mottaghy, F. M., Lebedeva, E., Ozer, E., et al. (2010). A novel presenilin1 mutation (Q223R) associated with early onset Alzheimer's disease, dysarthria and spastic paraparesis and decreased Abeta levels in CSF. Eur. J. Neurol. 17, 631-633. doi: 10.1111/j.14681331.2009.02810.x

Wanngren, J., Lara, P., Ojemalm, K., Maioli, S., Moradi, N., Chen, L., et al. (2014). Changed membrane integration and catalytic site conformation are two mechanisms behind the increased Abeta42/Abeta40 ratio by presenilin 1 familial Alzheimer-linked mutations. FEBS Open. Biol. 4, 393-406. doi: 10.1016/j.fob.2014.04.006
Wolfe, M. S., Xia, W., Ostaszewski, B. L., Diehl, T. S., Kimberly, W. T., and Selkoe, D. J. (1999). Two transmembrane aspartates in presenilin-1 required for presenilin endoproteolysis and gamma-secretase activity. Nature 398, 513-517. doi: 10.1038/19077

Wu, L., Rosa-Neto, P., Hsiung, G. Y., Sadovnick, A. D., Masellis, M., Black, S. E., et al. (2012). Early-onset familial Alzheimer's disease (EOFAD). Can. J. Neurol. Sci. 39, 436-445. doi: 10.1017/S03171671000 13949

Yu, G., Nishimura, M., Arawaka, S., Levitan, D., Zhang, L., Tandon, A., et al. (2000). Nicastrin modulates presenilin-mediated notch/glp-1 signal transduction and betaAPP processing. Nature 407, 48-54. doi: 10.1038/ 35024009

Conflict of Interest Statement: The authors declare that the research was conducted in the absence of any commercial or financial relationships that could be construed as a potential conflict of interest.

Copyright (c) 2016 Li, Liu, Qiu, Ren, Dai, Deng and Qing. This is an open-access article distributed under the terms of the Creative Commons Attribution License (CC BY). The use, distribution or reproduction in other forums is permitted, provided the original author(s) or licensor are credited and that the original publication in this journal is cited, in accordance with accepted academic practice. No use, distribution or reproduction is permitted which does not comply with these terms. 